Check for updates

Cite this: RSC Adv., 2018, 8, 37286

Received 24th July 2018

Accepted 17th October 2018

DOI: 10.1039/c8ra06252h

rsc.li/rsc-advances

\section{An improved HPLC-MS/MS method for simultaneous quantification of propranolol and its two phase I metabolites in plasma of infants with hemangioma and its application to a comparative study of plasma concentrations}

\author{
Huan He, $,^{\mathrm{a}} \mathrm{Li} \mathrm{Li}, \hat{\dagger}^{\mathrm{b}}$ Libo Zhao, ${ }^{a}$ Ning Sun, ${ }^{a}$ Meng Zhang, ${ }^{a}$ Ying Cheng, ${ }^{\mathrm{b}}$ Lu Yu, \\ Lin $\mathrm{Ma}^{* \mathrm{~b}}$ and Xiaoling Wang (D) *a
}

Propranolol is now a preferred treatment for infantile hemangioma. However, there are no published papers on the metabolism and concentrations of propranolol in the plasma of infants with hemangioma. In the present study, a sensitive, simple and reliable method was developed and validated for the simultaneous quantification of propranolol and its metabolites 4-hydroxypropranolol (M1) and $N$ desisopropylpropranolol (M2) in infants' plasma for the first time by using liquid chromatography-tandem mass spectrometry (LC-MS/MS). A volume of $100 \mu \mathrm{L}$ plasma was prepared by one-step protein precipitation with acetonitrile $(300 \mu \mathrm{L})$, followed by its separation on an Hypersil GOLD $\mathrm{C}_{18}$ column maintained at $40{ }^{\circ} \mathrm{C}$ with gradient mobile phase consisting of $0.1 \%$ formic acid aqueous solution and acetonitrile at a flow rate of $0.3 \mathrm{~mL} \mathrm{~min}^{-1}$. The quantification was performed via multiple reaction monitoring (MRM) by a triple quadrupole mass spectrometer under positive electrospray ionization (ESI) mode. Bisoprolol was chosen as the internal standard. The method was validated to demonstrate its selectivity, linearity, accuracy, precision, recovery, matrix effect and stability. The matrix-matched calibration curves for propranolol ranging from 1 to $500 \mathrm{ng} \mathrm{mL}^{-1}$, for $\mathrm{M} 1$ ranging from 0.2 to $100 \mathrm{ng}$ $\mathrm{mL}^{-1}$ and for $\mathrm{M} 2$ ranging from 0.2 to $100 \mathrm{ng} \mathrm{mL}^{-1}$ were all linear, with correlation coefficients calculated using weighted $\left(1 / x^{2}\right)$ least square linear regression analysis. The lower limits of quantification (LLOQs) were $1 \mathrm{ng} \mathrm{mL} L^{-1}, 0.2 \mathrm{ng} \mathrm{mL}^{-1}$ and $0.2 \mathrm{ng} \mathrm{mL}^{-1}$ for propranolol, $\mathrm{M} 1$ and $\mathrm{M} 2$, respectively. The intra-day and inter-day precisions were less than $7.1 \%$ and relative errors were all less than $9.8 \%$. This validated method was successfully applied to quantify the concentrations of propranolol and its metabolites 4hydroxypropranolol (M1) and N-desisopropylpropranolol (M2) in the plasma of infants with hemangioma after oral administration of different doses of propranolol for the first time.

\section{Introduction}

Infantile hemangioma ( $\mathrm{IH})$ is one of the common benign tumors that occurs during infancy, with a prevalence rate of around $4.5 \%{ }^{1}{ }^{1}$ The high-risk $\mathrm{IH}$ cases including life-threatening $\mathrm{IH}$, function-threatening $\mathrm{IH}$, severe ulceration and bleeding or risk of disfigurement require systemic therapy. ${ }^{1-3}$ Since the discovery of the therapeutic effects of propranolol on $\mathrm{IH}$ in 2008 , the treatment of IH has gone through a huge revolution. ${ }^{4}$

${ }^{a}$ Clinical Research Center, Beijing Children's Hospital, Capital Medical University, National Center for Children's Health, Beijing 100045, China. E-mail: eyjdb6380@ 163.com

${ }^{b}$ Department of Dermatology, Beijing Children's Hospital, Capital Medical University, National Center for Children's Health, Beijing 100045, China. E-mail: bch_maleen@ aliyun.com

$\dagger$ These authors contributed equally to this work.
Oral propranolol has become the preferred treatment for IH, and should be administered as early as possible to achieve better results. ${ }^{1,3,5}$ Several reviews and meta-analyses have demonstrated the effectiveness of oral propranolol and consensus conferences have made suggestions about initiation and use of propranolol for $\mathrm{IH}^{3,6,7}$ Studies have shown that the mechanisms of propranolol in the treatment of IH were (a) incorporated inhibition angiogenesis by reducing the level of angiogenic factors and (b) induction of cell apoptosis. ${ }^{8-10}$

Propranolol, a non-selective $\beta$-blocker, is widely metabolized in human liver into phase I and phase II metabolites. The phase I metabolites are formed by the way of naphthalene ring hydroxylation and $N$-dealkylation of the isopropylamine side chain. The phase II metabolite is formed by side chain $O$-glucuronidation. Propranolol is metabolized mainly by the P450 enzyme CYP2D6 to 4-hydroxypropranolol (M1), which has been reported as a major metabolite in humans. ${ }^{11-13}$ Propranolol is 
also metabolized by P450 enzyme CYP1A2 to $N$-desisopropylpropranolol (M2). ${ }^{12}$ However, the metabolism of propranolol in infants with hemangioma has not been studied so far. Propranolol is used in the treatment of $\mathrm{IH}$ and it is inevitable to study the exposed quantities of propranolol and its metabolites for further research and illumination of the relationships between dosage, safety, efficacy and exposure.

For the determination of propranolol, 4-hydroxypropranolol and $N$-desisopropylpropranolol in biological fluids, highperformance liquid chromatographic (HPLC) and liquid chromatography coupled with mass spectrometry (MS) methods have already been reported. ${ }^{13-17}$ However, the existing HPLC methods were complicated and excess plasma was used. In contrast, the LC-MS/MS method attracted our attention for the determination of propranolol and 4-hydroxypropranolol because the minimum detectable concentration using this method was $0.2 \mathrm{ng} \mathrm{mL}{ }^{-1} .^{13}$ However, this method was not able to simultaneously detect $N$ desisopropylpropranolol, and the usage volume of plasma is 300 $\mu \mathrm{L}$ with complex sample processing. Furthermore, none of the reported methods were applied in infants' plasma. Considering all the subjects participating in this research are infants with hemangioma and plasma concentrations of propranolol and its metabolites M1 and M2 that are all present as low levels (ng $\mathrm{mL}^{-1}$ ) after oral administration, the quantitative method should use a low volume of plasma $(\leq 100 \mu \mathrm{L})$ with a relatively lower detectable concentration ( $\leq 1 \mathrm{ng} \mathrm{mL}^{-1}$ ). Hence, an enhanced, highly accurate and sensitive analytical method is needed for the simultaneous quantification of propranolol and its metabolites M1 and M2.

In this study, a simple, sensitive and reliable liquid chromatography-tandem mass spectrometry (LC-MS/MS) method was developed and validated for the simultaneous quantification of propranolol and its metabolites M1 and M2. The validated LC-MS/MS method was successfully applied to determine the concentrations of propranolol and its metabolites M1 and M2 in the plasma of infants with hemangioma after oral administration of multiple doses of propranolol. In addition, the comparison of plasma concentrations at different dose was performed for the first time.

\section{Experimental}

\subsection{Chemicals and reagents}

Reference standards of propranolol hydrochloride $(\geq 98.5 \%$ purity), 4-hydroxypropranolol hydrochloride ( $\geq 98.5 \%$ purity), $N$-desisopropylpropranolol ( $\geq 97.5 \%$ purity) and bisoprolol hemifumarate salt ( $\geq 98 \%$ purity, Internal standard, IS) were all purchased from Sigma-Aldrich (St. Louis, MO, USA). HPLC grade acetonitrile and methanol were obtained from Fisher Scientific (Pittsburgh, USA). All other chemicals were of analytical grade. Distilled water was purchased from Watsons (Hong Kong, China).

\subsection{Instrumentation and analytical conditions}

A Shimadzu LC-20A HPLC system (Shimadzu, Japan) equipped with two LC-20ADXR pumps, an SIL-20ACHT autosampler, a CTO-20A column oven and a DGU-20A3R degasser (Shimadzu, Kyoto, Japan) was used for chromatographic separation. Mass spectrometric detection was performed on an AB Sciex Qtrap 5500 System (Applied Biosystems-Sciex, Toronto, Canada) equipped with a Turbo Ion Spray Source. Analyst software version 1.6.2 (Applied Biosystems) was used to control the LCMS/MS system and for data acquisition.

Samples were separated on a Hypersil GOLD C $18(150 \times 2.1$ mm, $5 \mu \mathrm{m}$; Thermo Fisher Scientific Inc., Boston, USA) maintained at $40{ }^{\circ} \mathrm{C}$, with gradient elution. The mobile phase consisting of $0.1 \%$ formic acid aqueous solution (A) and acetonitrile (B) was run at a flow rate of $0.3 \mathrm{~mL} \mathrm{~min}^{-1}$. The gradient was as follows: $0 \mathrm{~min}, 10 \% \mathrm{~B} ; 2 \mathrm{~min}, 70 \% \mathrm{~B} ; 6 \mathrm{~min}$, $70 \% \mathrm{~B} ; 6.1 \mathrm{~min} 10 \% \mathrm{~B} ; 9.5 \mathrm{~min}, 10 \% \mathrm{~B}$. The autosampler temperature was set at $4{ }^{\circ} \mathrm{C}$ and the injection volume was $10 \mu \mathrm{L}$.

The mass spectrometer was operated in positive electrospray ionization (ESI) mode and the quantification was performed via multiple reaction monitoring (MRM). The source parameters were as follows: ion spray voltage, $5500 \mathrm{~V}$; capillary temperature, $500{ }^{\circ} \mathrm{C}$; curtain gas (CUR), nebulizer gas (GS1) and turbo gas (GS2) pressure were 35, 50 and 50 psi, respectively; collision activation dissociation (CAD), medium. The precursor to product ion transitions were as follows: $\mathrm{m} / \mathrm{z}$ $260.0 \rightarrow 116.0$ for propranolol, with the declustering potential (DP), collision energy (CE) and collision cell exit potential (CXP) set to 75, 25 and $13 \mathrm{~V}$, respectively; $\mathrm{m} / \mathrm{z} 276.1 \rightarrow 173.0$ for M1 with the DP, CE and CXP set to 94, 25 and $20 \mathrm{~V}$, respectively; $m / z 218.0 \rightarrow 74.0$ for $\mathrm{M} 2$ with the DP, CE and CXP set to 81,18 and $16 \mathrm{~V}$, respectively; and $m / z 326.1 \rightarrow 116.0$ for the IS with the DP, CE and CXP set to 108, 24 and $19 \mathrm{~V}$, respectively.

\subsection{Preparation of standard solutions, calibration samples and quality control (QC) samples}

Stock solutions of propranolol, M1 and M2 were prepared in methanol at $0.1 \mathrm{mg} \mathrm{mL}^{-1}$. Then, the mixed stock solutions were serially diluted in methanol to obtain the calibration standard solutions containing propranolol (5, 10, 25, 100, 250, 500, 1000, and $\left.2500 \mathrm{ng} \mathrm{mL}{ }^{-1}\right)$, M1 and M2 (1, 2, 5, 20, 50, 100, 200, and 500 $\left.\mathrm{ng} \mathrm{mL^{-1 }}\right)$. QC solutions containing propranolol $(5,10,500$, and $\left.2000 \mathrm{ng} \mathrm{mL}{ }^{-1}\right)$, M1 and M2 (1, 2, 100, and $400 \mathrm{ng} \mathrm{mL}^{-1}$ ) were also prepared as described above. The IS stock solution was prepared at a concentration of $0.1 \mathrm{mg} \mathrm{mL}^{-1}$ in methanol and

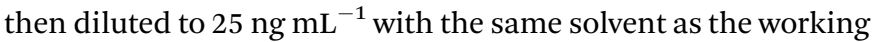
solution. All solutions were stored at $-40^{\circ} \mathrm{C}$.

Plasma calibration standards were prepared by adding $20 \mu \mathrm{L}$ standard working solutions into $100 \mu \mathrm{L}$ blank children's plasma to obtain the desired concentrations of propranolol $(1,2,5,20$, $50,100,200$, and $\left.500 \mathrm{ng} \mathrm{mL} \mathrm{m}^{-1}\right)$, M1 and M2 (0.2, 0.4 , 1, 4, 10, 20, 40 , and $\left.100 \mathrm{ng} \mathrm{mL}{ }^{-1}\right)$. IS working solution $(20 \mu \mathrm{L})$ was added to the plasma and then, the mixture was subjected to protein precipitation, followed by LC-MS/MS analysis (Section 2.4). The four levels of QC samples at lower limit of quantification (LLOQ), low QC (LQC), medium QC (MQC) and high QC (HQC) for propranolol $\left(1,2,100\right.$, and $\left.400 \mathrm{ng} \mathrm{mL}^{-1}\right)$, M1 and M2 (0.2, $0.4,20$, and $80 \mathrm{ng} \mathrm{mL}^{-1}$ ) were prepared in the same way. 


\subsection{Sample preparation}

Children plasma $(100 \mu \mathrm{L})$ was mixed with $20 \mu \mathrm{L}$ IS working solution $\left(25 \mathrm{ng} \mathrm{mL}^{-1}\right)$ and $20 \mu \mathrm{L}$ methanol, followed by addition of $300 \mu \mathrm{L}$ acetonitrile. The mixtures were vortexed for $1 \mathrm{~min}$ and centrifuged at $13500 \mathrm{rpm}$ at $4{ }^{\circ} \mathrm{C}$ for $10 \mathrm{~min}$ to remove the precipitate. Then, $10 \mu \mathrm{L}$ of the supernatant was injected into the LC-MS/MS system for analysis of M1 and M2. Another $100 \mu \mathrm{L}$ of the supernatant was transferred to $1.5 \mathrm{~mL}$ tubes and diluted by adding $600 \mu \mathrm{L}$ acetonitrile Then, $10 \mu \mathrm{L}$ of the resulting solution was injected into the LC-MS/MS system for analysis of propranolol.

\subsection{Method validation}

The selectivity, linearity, lower limit of quantification, precision and accuracy, extraction recovery, matrix effect and stability of this method were validated.

To evaluate the selectivity of the method, six different individual children plasma were applied by comparing the chromatograms of blank plasma samples and blank plasma spiked with analytes and IS at the LLOQ level.

The plasma calibration curves were constructed by plotting the peak area ratios (analyte/IS) versus analyte concentrations of the eight calibration standards (described in 2.3) and evaluated in three separate times. The peak area ratios $(y)$ and concentrations of each analyte $(x)$ were analyzed by a weighted $\left(1 / x^{2}\right)$ least squares linear regression to calculate the calibration equation and correlation coefficients. The accuracies of the calculated concentrations for each calibration curves should be within $\pm 15 \%$, while the LLOQ level can be within $20 \%$.

The intra- and inter-day precision and accuracy were evaluated by analyzing six replicate QC samples at four concentration levels (LLOQ, LQC, MQC and HQC) on three consecutive days. Relative standard deviation (RSD, \%) and relative error (RE, \%) were calculated to express the precision and accuracy, respectively. For the LQC, MQC and HQC samples, the RSD and RE values should be within $\pm 15 \%$. For the LLOQ samples, the precision (RSD) and accuracy (RE) were both required within $20 \%$.

The recoveries of propranolol, M1, M2 and IS were determined at three QC concentrations (LQC, MQC and HQC) by comparing the peak areas of QC samples with the peak areas of samples at the same concentration spiked with work solutions after plasma protein precipitation. To investigate the matrix effect, six different individual children plasma samples were used to obtain the matrix. The matrix factors (MFs) of propranolol, M1, M2 and IS were regarded as peak area ratios of analytes and IS dissolved in matrix to analytes and IS dissolved in water at three concentrations. The IS-normalized MFs were calculated by dividing the MF of analytes by the MF of IS, with an RSD value of less than $15 \%$. The hemolytic matrix effect was conducted by using the $2 \%$ hemolytic plasma. The operation approach was the same as the validation of matrix effect.

The stabilities of propranolol, M1 and M2 in the infants' plasma were assessed by the analysis of six replicate QC samples at three concentrations in different conditions. The QC samples exposed to room temperature $\left(20^{\circ} \mathrm{C}\right)$ for $6 \mathrm{~h}$ and stored at
$-80{ }^{\circ} \mathrm{C}$ for 31 days were chosen to investigate the stability of analytes at room temperature (encountered during the plasma preparation) and long-term stability, respectively. Freeze-thaw stability was tested by performing three cycles of freezing $(-80$ $\left.{ }^{\circ} \mathrm{C}\right)$ and thawing $\left(20^{\circ} \mathrm{C}\right)$. The stabilities of the three analytes in the processed samples stored in an autosampler at $4{ }^{\circ} \mathrm{C}$ for $24 \mathrm{~h}$ were also evaluated. In the stability test, deviations were acceptable within $\pm 15 \%$.

\subsection{Application of the method}

The validated method was applied to analyze plasma samples obtained from infants with hemangioma after oral propranolol treatment. The experiment was performed in accordance with the guidelines of Declaration of Helsinki and Good Clinical Practice. The study was approved by the Ethics Committees of Beijing Children's Hospital. Parents' informed consents were obtained for human participants of this study. The infants with hemangioma who were enrolled in this study at the department of dermatology of Beijing Children's Hospital received a 3 daytreatment of propranolol with the dose of 1.5 or $2 \mathrm{mg} \mathrm{kg} \mathrm{kg}^{-1}$ $\mathrm{d}^{-1}$. On the first day, the patients were administrated half level of the dose, followed by the normal dose. Propranolol was taken orally twice a day at 8:00 am and 20:00 pm. It was dissolved in water and then given to the infants. Only one blood sample was taken from each patient as the infants with hemangioma were all below 1 year old. Blood samples were collected at $1 \mathrm{~h}$ predose or $1 \mathrm{~h}$ post-dose during the morning on the third day. Hence, the amount of time that elapsed between dose administration and blood sample acquisition was 11 hours for the predose group and 1 hour for the post-dose group. Samples were centrifuged at $3000 \mathrm{rpm}$ for $10 \mathrm{~min}$ and plasma were separated and stored at $-80{ }^{\circ} \mathrm{C}$ until analysis.

\section{Results and discussion}

\subsection{LC-MS/MS method development}

For the optimization of mass spectrometric conditions, each of the standard solutions of the compounds was continuously infused into the mass spectrometer by using a syringe infusion pump. Due to the existence of basic amino groups in the structures, both the three analytes and IS showed strong signals in the positive ionization mode under an ESI source. The MRM mode was selected for quantification. The full-scan product ion mass spectra and fragmentation pathways of the analytes and IS are shown in Fig. 1. The most abundant ion fragment $[\mathrm{M}+\mathrm{H}]^{+}$ was selected in the MRM transitions. Therefore, the precursor to product ion reactions were $m / z 260.0 \rightarrow 116.0$ for propranolol, $m / z 276.1 \rightarrow 173.0$ for M1, $m / z 218.0 \rightarrow 74.0$ for M2 and $m /$ $z 326.1 \rightarrow 116.0$ for IS. Other MS parameters such as ion spray voltage, capillary temperature, curtain gas pressure, nebulizer gas pressure, turbo gas pressure, collision activation dissociation and collision energy were further optimized and listed in Section 2.2.

During the optimization of chromatographic conditions, different columns and mobile phases were tested to achieve separated peak shapes, efficient ionization and appropriate 

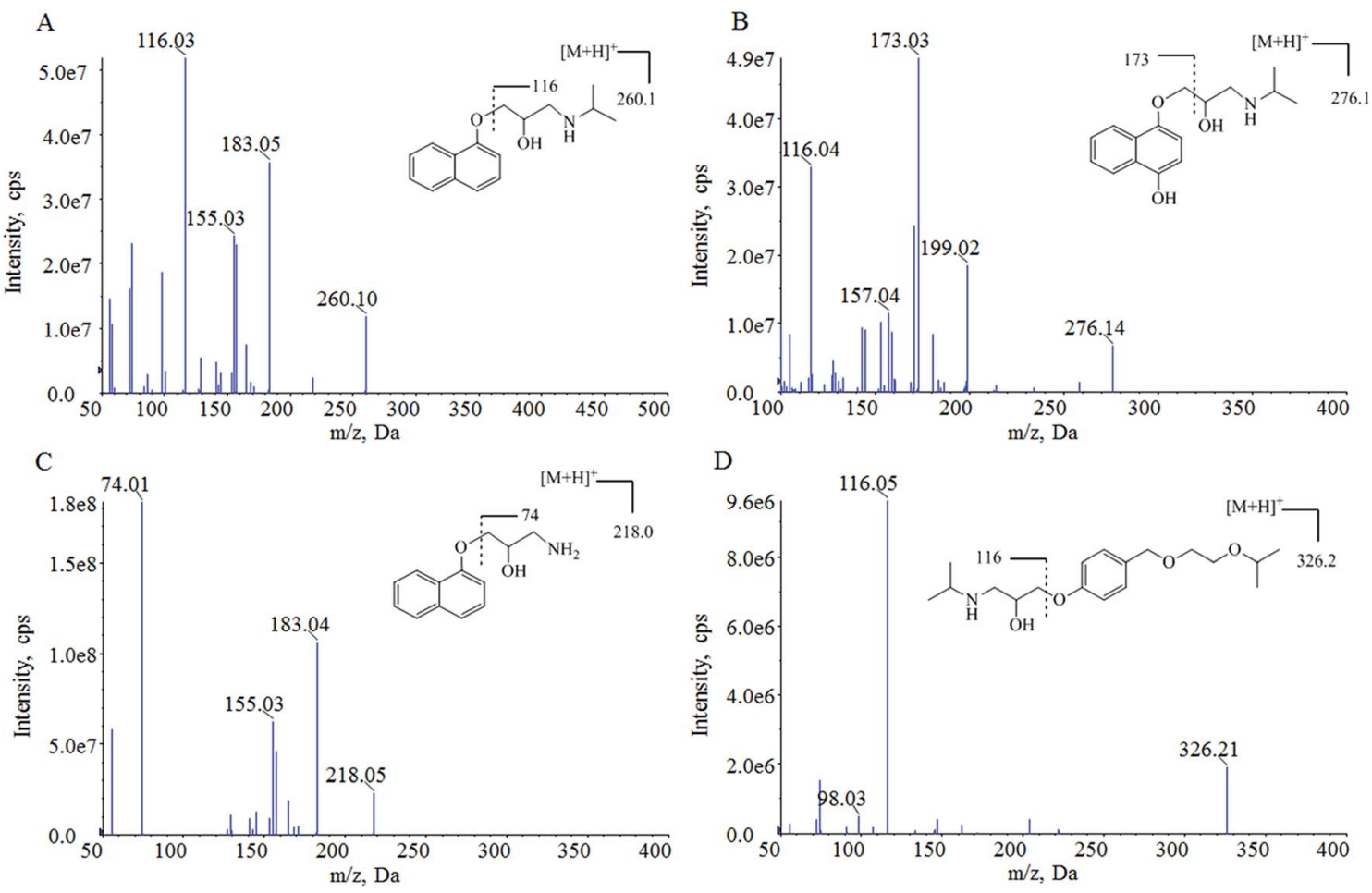

Fig. 1 The product ion spectra and structures of propranolol (A), M1 (B), M2 (C) and IS (D).

chromatographic running time. Finally, acetonitrile and water with $0.1 \%$ formic acid was chosen as the mobile phase for use on a Hypersil GOLD $\mathrm{C}_{18}$ column in a gradient elution program. Under these chromatographic conditions, the retention times of propranolol, M1, M2 and IS were 4.22, 3.98, 4.08, and $4.16 \mathrm{~min}$, respectively. The analytes and IS were separated from endogenous interferences. The typical chromatograms are shown in Fig. 2.

Protein precipitation of the children's plasma $(100 \mu \mathrm{L})$ with trifold acetonitrile was utilized as the sample preparation method in this assay, and showed good performance in terms of clean extracts and high recovery. The matrix effect trials demonstrated that no significant signal suppression or enhancement was found. This simple and efficient sample preparation method could meet the requirement of analysis of thousands of plasma samples during clinical trials.

A suitable internal standard is usually needed in LC-MS/MS analysis to eliminate the effects from matrix and the sample preparation efficiency. In this study, bisoprolol, an available structural analog of propranolol, was chosen as the IS. It displayed similar chromatographic behavior and high extraction efficiency with the three analytes. Furthermore, no interference of IS was found from propranolol-, M1-, M2-containing samples.

\subsection{Method validation}

3.2.1. Selectivity. The typical chromatograms of blank plasma and blank plasma spiked with propranolol, M1, M2 at the LLOQ level $\left(1 \mathrm{ng} \mathrm{mL}{ }^{-1}\right.$ for propranolol and $0.2 \mathrm{ng} \mathrm{mL}^{-1}$ for M1 and M2) and IS (5 $\mathrm{ng} \mathrm{mL}^{-1}$ ) and the plasma of the infant obtained at $1 \mathrm{~h}$ after oral administration of $2 \mathrm{mg} \mathrm{kg}{ }^{-1}$ propranolol are shown in Fig. 2. There are no significant endogenous interference peaks at the retention times of propranolol (4.22 min), M1 (3.98 $\mathrm{min}), \mathrm{M} 2$ (4.08 $\mathrm{min}$ ), and IS (4.16 min), suggesting good selectivity of the developed method.

3.2.2. Linearity and LLOQ. The standard calibration curves for propranolol ranging from 1 to $500 \mathrm{ng} \mathrm{mL}{ }^{-1}$, for M1 ranging. from 0.2 to $100 \mathrm{ng} \mathrm{mL}^{-1}$ and for M2 ranging from 0.2 to $100 \mathrm{ng}$ $\mathrm{mL}^{-1}$ were linear, with correlation coefficients calculated by using weighted $\left(1 / x^{2}\right)$ least square linear regression analysis. The typical linear regression equations were as follows: propranolol, $y=0.053 x+0.0112(r=0.9992) ;$ M1, $y=0.0198 x+$ $0.000344(r=0.9989)$; M2, $y=0.0217 x+0.0000049(r=0.9993)$. Standard deviations of the three calibration curves for each analyte were evaluated with REs of the calculated concentrations, and all the REs were within $\pm 15 \%$. The LLOQs of propranolol, M1 and M2 were $1 \mathrm{ng} \mathrm{mL} L^{-1}, 0.2 \mathrm{ng} \mathrm{mL}^{-1}$ and 0.2 $\mathrm{ng} \mathrm{mL} \mathrm{mL}^{-1}$, respectively, with RE and RSD less than $20 \%$, obtained in three separate validation batches (Table 1). These concentration ranges and LLOQs were suitable and sufficiently sensitive for concentration detection in the plasma of infants with hemangioma.

3.2.3. Precision and accuracy. The intra-day and inter-day precision and accuracy values are shown in Table 1. For the three QC samples (LQC, MQC and HQC), the intra-day precision were less than $5.5 \%$, and the inter-day precision were less than $5.2 \%$. The mean REs ranged between $-7.5 \%$ and $8.0 \%$. These results indicated that the developed method was accurate and repeatable.

3.2.4. Recovery and matrix effect. As shown in Table 2 , the mean recoveries of propranolol, M1 and M2 at three levels of QC samples were all higher than $92.45 \%$. The recoveries of IS were 
A
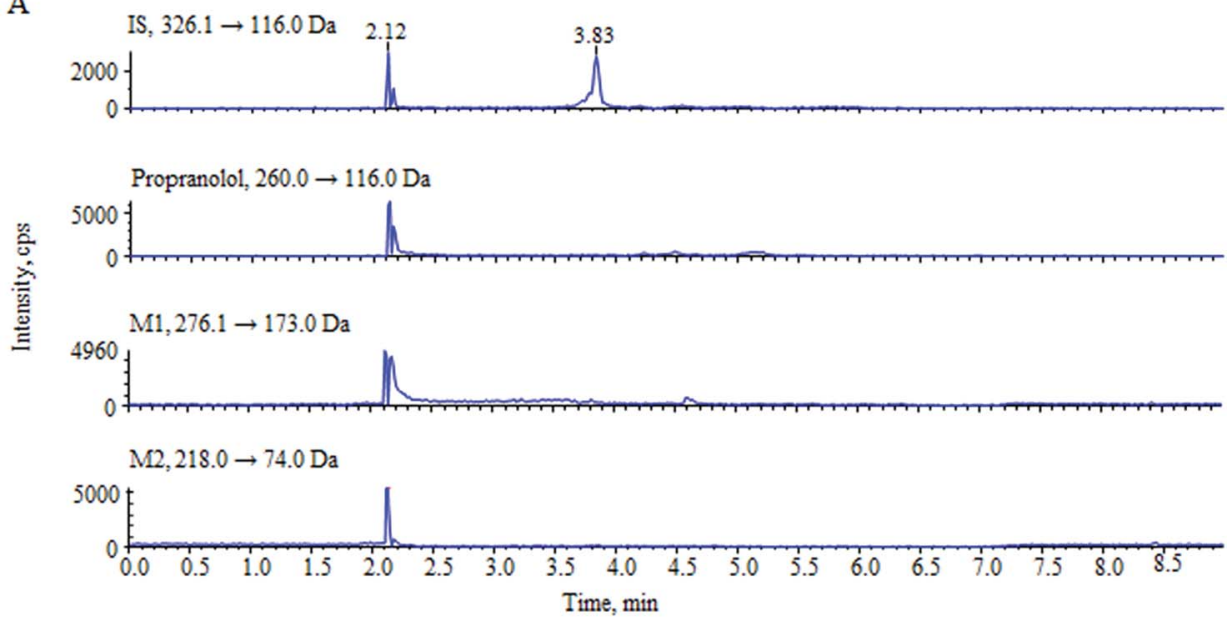

B
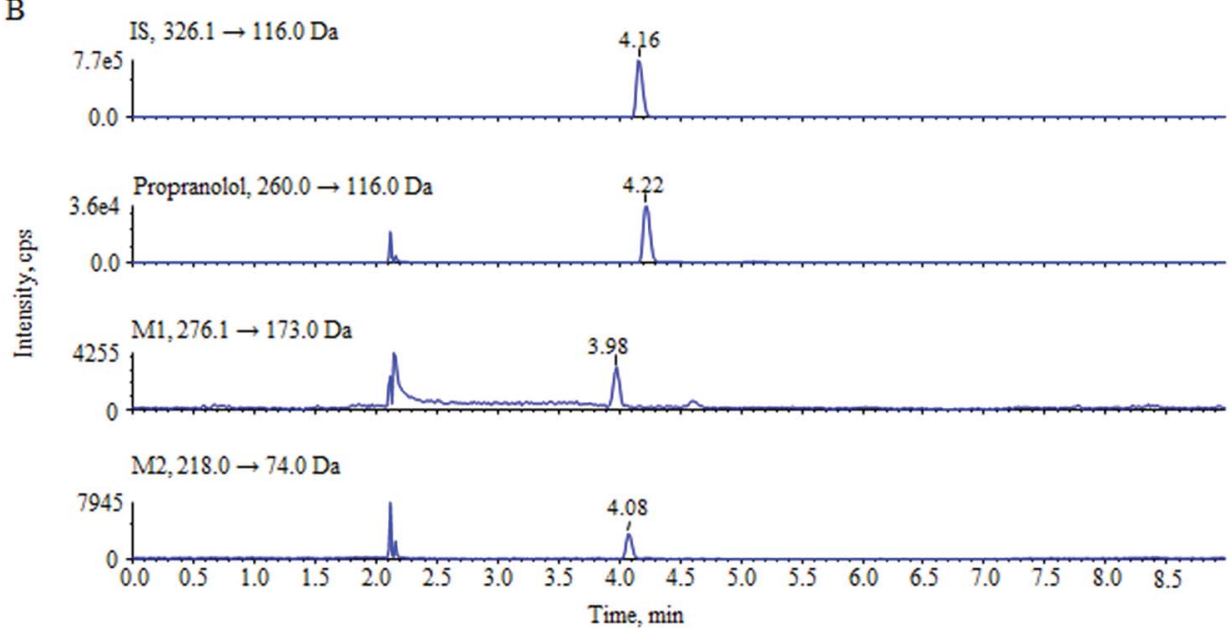

$\mathrm{C}$
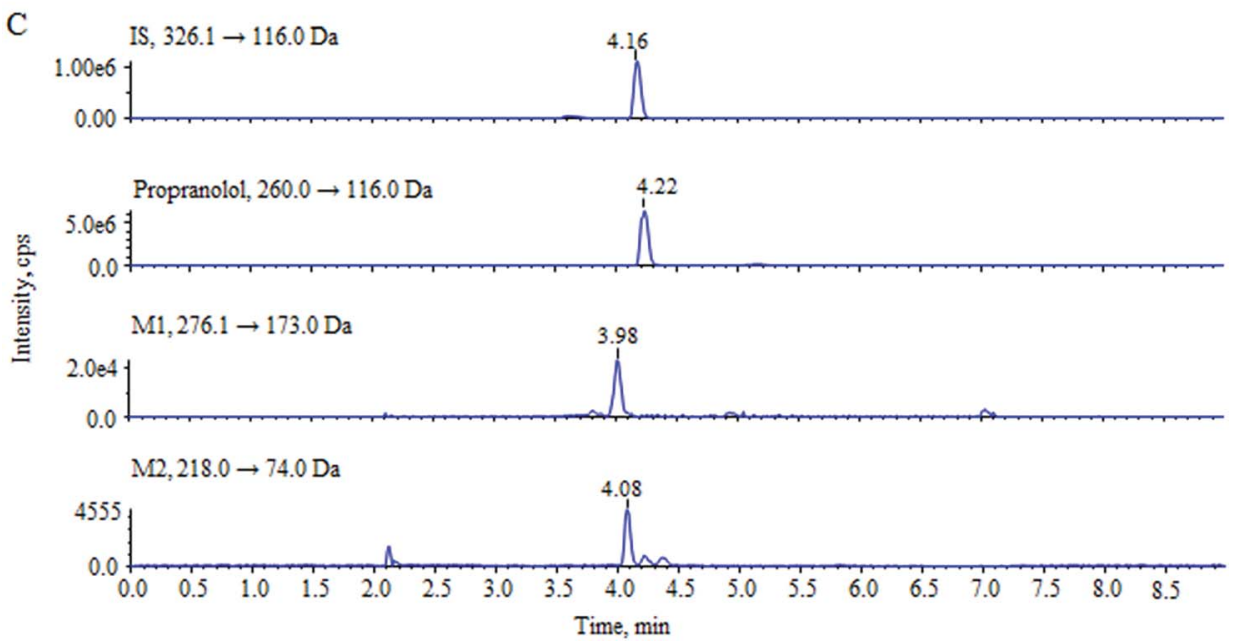

Fig. 2 The representative MRM chromatograms of propranolol, M1, M2 and IS in plasma samples: (A) blank plasma sample; (B) blank plasma spiked with LLOQ level standard solution and IS; (C) plasma sample collected at $1 \mathrm{~h}$ post-dose from a patient with infantile hemangioma treatment with $2 \mathrm{mg} \mathrm{kg}^{-1} \mathrm{~d}^{-1}$ propranolol.

99.61\% for propranolol detection and $96.73 \%$ for M1 and M2 detection. These results indicated that more than $92.45 \%$ of the analytes and IS were recovered after sample preparation by protein precipitation.
Matrix-matched calibration was employed because matrix effect was observed when using external calibration. Matrix effect of the matrix-matched calibration was evaluated. The results are summarized in Table 2. IS-normalized matrix 
Table 1 The intra- and inter-day precisions and accuracies of propranolol, M1 and M2 in children's plasma $(n=6$ for intra-day precision, $n=3$ for inter-day precision) ${ }^{a}$

\begin{tabular}{|c|c|c|c|c|c|c|c|c|}
\hline Analyte & Level & $\begin{array}{l}\text { Spiked-concentration } \\
\left(\text { ng } \mathrm{mL}^{-1}\right)\end{array}$ & $\begin{array}{l}\text { Measured } \\
(\text { mean } \pm \mathrm{SD})\end{array}$ & $\begin{array}{l}\text { Precision } \\
\text { (RSD\%) }\end{array}$ & $\begin{array}{l}\text { Accuracy } \\
(\mathrm{RE} \%)\end{array}$ & $\begin{array}{l}\text { Measured } \\
(\text { mean } \pm \mathrm{SD})\end{array}$ & $\begin{array}{l}\text { Precision } \\
\text { (RSD\%) }\end{array}$ & $\begin{array}{l}\text { Accuracy } \\
(\mathrm{RE} \%)\end{array}$ \\
\hline \multirow[t]{3}{*}{ Propranolol } & LLOQ & 1 & $0.90 \pm 0.02$ & 2.6 & -9.8 & $0.92 \pm 0.07$ & 7.1 & -7.5 \\
\hline & LQC & 2 & $1.9 \pm 0.1$ & 5.5 & -3.6 & $1.94 \pm 0.09$ & 4.5 & -3.0 \\
\hline & MQC & 100 & $102 \pm 2$ & 2.1 & 1.8 & $99 \pm 3$ & 2.7 & -0.9 \\
\hline & LQC & 0.4 & $0.42 \pm 0.02$ & 3.8 & 4.5 & $0.40 \pm 0.02$ & 3.9 & 1.1 \\
\hline & MQC & 20 & $21 \pm 0.7$ & 3.0 & 7.0 & $20 \pm 1$ & 5.2 & 1.1 \\
\hline & HQC & 80 & $86 \pm 2$ & 2.2 & 8.0 & $82 \pm 4$ & 4.8 & 2.4 \\
\hline \multirow[t]{3}{*}{ M2 } & LLOQ & 0.2 & $0.22 \pm 0.01$ & 6.0 & 7.6 & $0.20 \pm 0.01$ & 5.3 & 1.5 \\
\hline & LQC & 0.4 & $0.41 \pm 0.01$ & 2.8 & 2.3 & $0.40 \pm 0.02$ & 4.3 & -0.6 \\
\hline & MQC & 20 & $19.7 \pm 0.3$ & 1.7 & -1.4 & $19.4 \pm 0.5$ & 2.7 & -3.0 \\
\hline
\end{tabular}

${ }^{a}$ LLOQ: lower limit of quantification; LQC: low quality control; MQC: medium quality control; HQC: high quality control; RE: relative error.

Table 2 Recovery and matrix effect of propranolol, M1 and M2 in children's plasma $(n=6)^{a}$

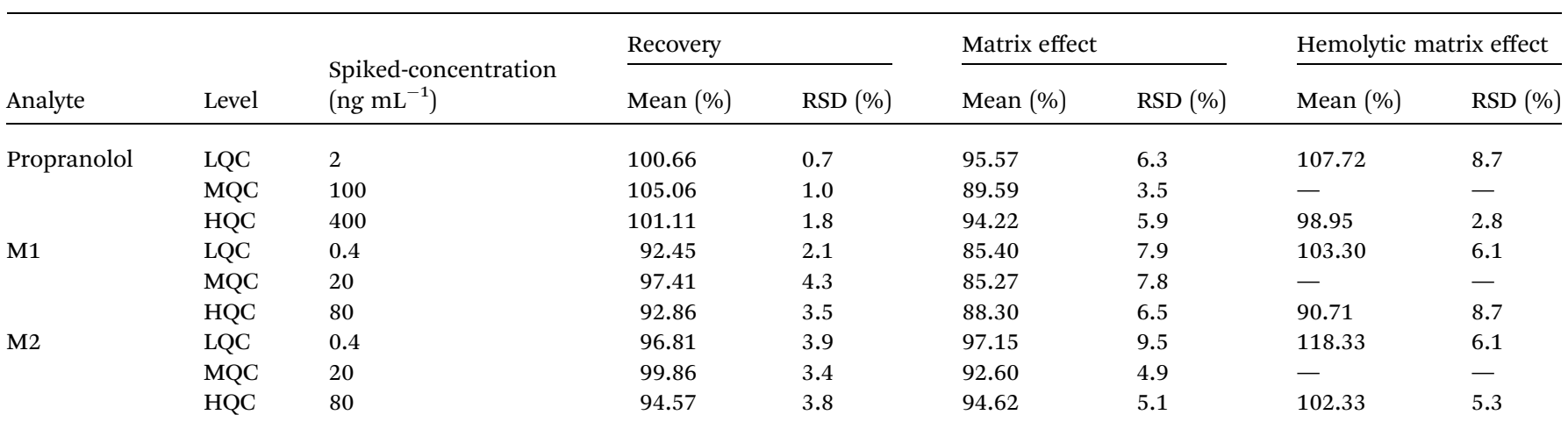

${ }^{a}$ LQC: low quality control; MQC: medium quality control; HQC: high quality control.

factors of propranolol, $\mathrm{M} 1$ and $\mathrm{M} 2$ at three levels of $\mathrm{QC}$ samples were between $85.27 \%$ and $97.15 \%$, with the RSD values from six lots of blank children's plasma samples being less than $9.5 \%$. Furthermore, the matrix effect of hemolytic plasma was also evaluated, and the IS-normalized matrix factors at LQC and HQC levels were between $90.71 \%$ and $118.33 \%$, respectively, with RSD values less than $8.7 \%$. Therefore, matrix effect of matrix matched calibration for this method was not observed.

3.2.5. Stability. The stability results of propranolol, M1 and M2 at different storage and processing conditions are presented in Table 3. The three analytes were stable in the children's plasma samples after storing at room temperature for $6 \mathrm{~h}$, at $-80{ }^{\circ} \mathrm{C}$ for at least 31 days, and after subjecting to three freeze-thaw cycles. In the processed samples, all of the analytes were also stable after storing in autosampler at $4{ }^{\circ} \mathrm{C}$ for $24 \mathrm{~h}$. The RSD and RE values were all within $\pm 15 \%$ for the stability test. These results indicated that the method showed good stability during routine sample analysis.

\subsection{Method application}

The validated LC-MS/MS method was used to detect the plasma concentrations of propranolol and its metabolites M1 and M2 in 207 infants with hemangioma after oral propranolol treatment. The clinical data are listed in Table 4 . The distributions of plasma concentrations of different doses and at different points are presented in box-and-whiskers plots in Fig. 3. The plots show that the concentrations of propranolol are significantly higher than those of M1 and M2. The average concentrations of M1 were three percent to seven percent of propranolol, while the average concentrations of M2 were 6 per thousand to 8 per thousand of propranolol. Furthermore, there are wide interindividual variations observed in Fig. 3. Based on the parameter that the volume of blood is about $80 \mathrm{~mL} \mathrm{~kg}^{-1}$ in an infant, ${ }^{18}$ the average percentage of the administered propranolol that is present in the blood at different times were estimated. When treated with propranolol at $1.5 \mathrm{mg} \mathrm{kg} \mathrm{kg}^{-1} \mathrm{~d}^{-1}$, the average percentage of propranolol in the infants' blood was $0.141 \%$ and $0.216 \%$ at the time of pre-dose and $1 \mathrm{~h}$ post-dose measurement, 
Table 3 Stability of propranolol, M1 and M2 in children's plasma at different conditions $(n=6)^{a}$

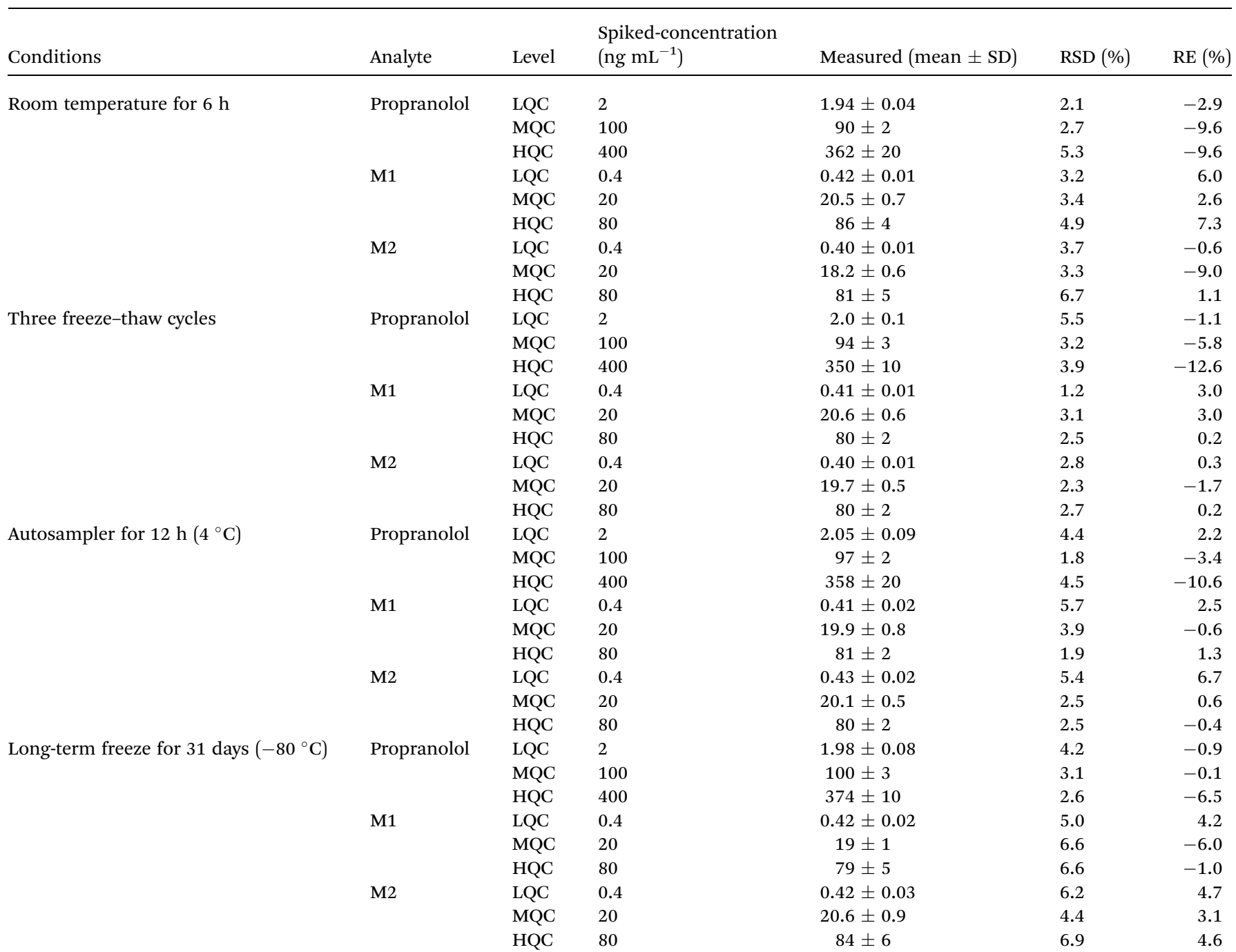

${ }^{a}$ LQC: low quality control; MQC: medium quality control; HQC: high quality control; RE: relative error.

Table 4 Clinical data of the study

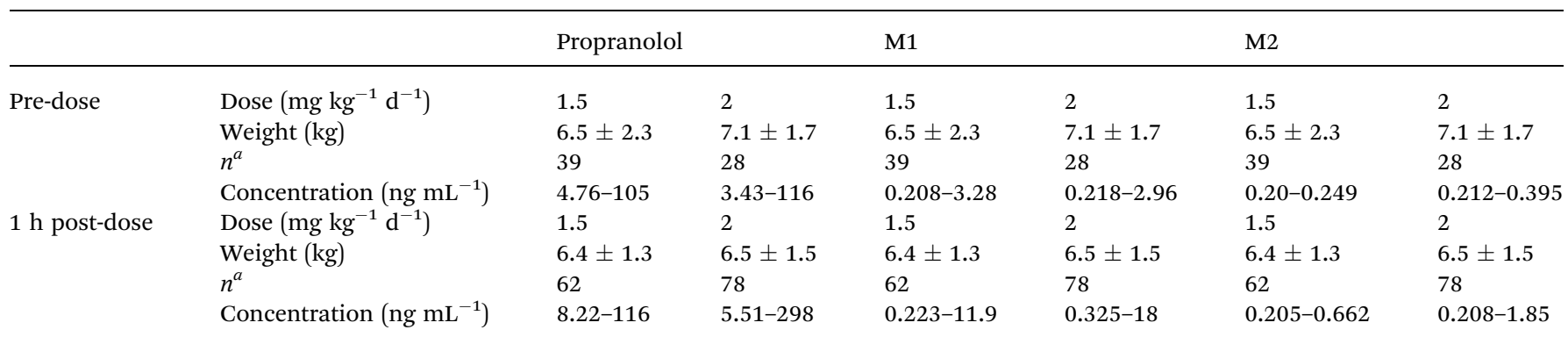

${ }^{a}$ Represent the number of patients in each group.

respectively. In the $2.0 \mathrm{mg} \mathrm{kg}^{-1} \mathrm{~d}^{-1}$ dose group, the percentage values were $0.127 \%$ and $0.269 \%$.

Statistical analysis was performed and the results are presented in Fig. 4. Undoubtedly, there are significant differences between the concentrations of pre-dose and $1 \mathrm{~h}$ post-dose for propranolol and M1, but not for M2. By comparing the concentrations of the dose $1.5 \mathrm{mg} \mathrm{kg}^{-1} \mathrm{~d}^{-1}$ and $2.0 \mathrm{mg} \mathrm{kg} \mathrm{kg}^{-1}$ $\mathrm{d}^{-1}$, statistical differences between the post-dose groups and no 

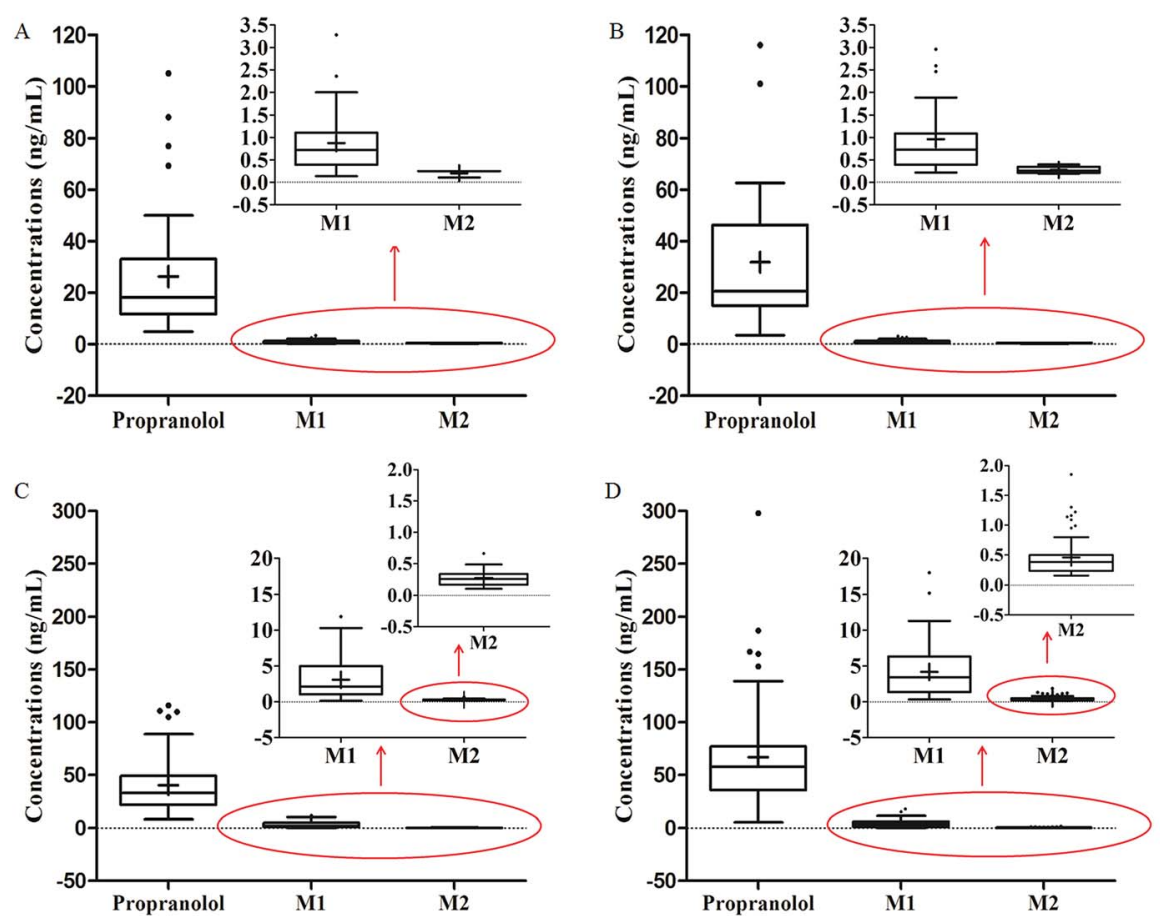

Fig. 3 The concentrations of propranolol and its metabolites $M 1$ and $M 2$ in the plasma of infants with $\mathrm{IH}$ : (A) the concentrations with a dose of $1.5 \mathrm{mg} \mathrm{kg}^{-1} \mathrm{~d}^{-1}$ at pre-dose; (B) the concentrations with a dose of $2 \mathrm{mg} \mathrm{kg}^{-1} \mathrm{~d}^{-1}$ at pre-dose; (C) the concentrations with a dose of $1.5 \mathrm{mg} \mathrm{kg}^{-1}$ $\mathrm{d}^{-1}$ at $1 \mathrm{~h}$ post-dose; (D) the concentrations with a dose of $2 \mathrm{mg} \mathrm{kg}^{-1} \mathrm{~d}^{-1}$ at $1 \mathrm{~h}$ post-dose. Data were presented in box-and-whiskers plots.
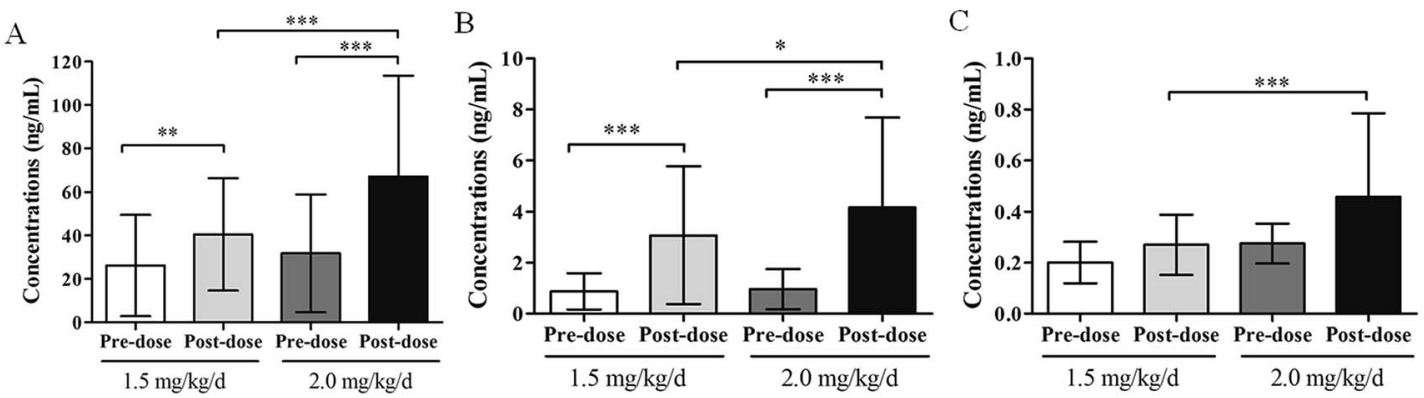

Fig. 4 The concentrations of propranolol and its metabolites M1 and M2 in plasma samples with different doses and at different time points: (A) the concentrations of propranolol; (B) the concentrations of $\mathrm{M} 1$; (C) the concentrations of $\mathrm{M} 2$. The bar charts are expressed as mean \pm standard deviation. The number of patients in each group were represented as $n . n=39$ for the group of dose of $1.5 \mathrm{mg} \mathrm{kg}^{-1} \mathrm{~d}^{-1}$ at pre-dose; $n=28$ for the group of $2 \mathrm{mg} \mathrm{kg}^{-1} \mathrm{~d}^{-1}$ dose at pre-dose; $n=62$ for the group of $1.5 \mathrm{mg} \mathrm{kg}^{-1} \mathrm{~d}^{-1}$ dose at $1 \mathrm{~h}$ post-dose; $n=78$ for the group of $2 \mathrm{mg} \mathrm{kg}^{-1} \mathrm{~d}^{-1}$ dose at $1 \mathrm{~h}$ post-dose; *** indicates $P<0.001, * *$ indicates $P<0.01, *$ indicates $P<0.05$.

significant difference between the pre-dose are observed. This result indicates that much more attention should be paid on the peak concentrations to study the relationships between the security and therapeutic drug concentration of different doses.

Furthermore, there are high standard deviations in the concentrations in infants with hemangioma, indicating that large inter-individual differences exist. These variations might be attributed to some factors that include age, weight, dosage regimens, liver function, and renal function. Further research such as developing a population pharmacokinetic model to study the sources of inter-individual variances of propranolol in infants with hemangioma is needed. This study found a phenomenon that the individual difference of propranolol concentration in treating infantile hemangioma was large and accumulated data for further research.

\section{Conclusion}

An improved LC-MS/MS method has been developed for the simultaneous determination of propranolol and its metabolites 4-hydroxypropranolol and $N$-desisopropylpropranolol in children's plasma. The proposed method has been fully validated and proved to be sensitive, simple and reliable. The LLOQs were $1 \mathrm{ng} \mathrm{mL}{ }^{-1}$ for propranolol and $0.2 \mathrm{ng} \mathrm{mL}^{-1}$ for its metabolites, 
which were suitable for the application of this method. The method showed recovery more than $92.45 \%$ by the simple protein precipitation procedure, and no matrix effects were observed due to the matrix-matched calibration. In this study, the method was successfully applied to quantify the concentrations of propranolol and its metabolites 4-hydroxypropranolol and $N$-desisopropylpropranolol in the plasma of infants with hemangioma after oral propranolol treatment at different doses for the first time. The statistic differences in plasma concentrations between the dose $1.5 \mathrm{mg} \mathrm{kg}^{-1} \mathrm{~d}^{-1}$ and $2.0 \mathrm{mg} \mathrm{kg}^{-1} \mathrm{~d}^{-1}$ in the $1 \mathrm{~h}$ post-dose group were different from those in the pre-dose group.

This is the first report about the quantification of propranolol and its metabolites in the plasma of infants with hemangioma, and it would lay a foundation for the study of population pharmacokinetics of propranolol in the infants with hemangioma.

\section{Conflicts of interest}

There are no conflicts to declare.

\section{Acknowledgements}

This study was supported by the Capital's Founds for Health Improvement and Research (2016-2-2093), Beijing Municipal Administration of Hospitals Incubating Program (PX2016014) and National science and technology major research project (2018ZX09721003).

\section{References}

1 C. Léauté-Labrèze, J. I. Harper and P. H. Hoeger, Lancet, 2017, 390, 85-94.

2 C. Léaute-Labrèze, O. Boccara, C. Degrugillier-Chopinet, J. Mazereeuw-Hautier, S. Prey, G. Lebbé, S. Gautier, V. Ortis, M. Lafon, A. Montagne, A. Delarue and J. J. Voisard, Pediatrics, 2016, 138, e20160353.

3 B. A. Drolet, P. C. Frommelt, S. L. Chamlin, A. Haggstrom, N. M. Bauman, Y. E. Chiu, R. H. Chun, M. C. Garzon,
K. E. Holland, L. Liberman, S. MacLellan-Tobert, A. J. Mancini, D. Metry, K. B. Puttgen, M. Seefeldt, R. Sidbury, K. M. Ward, F. Blei, E. Baselga, L. Cassidy, D. H. Darrow, S. Joachim, E. K. Kwon, K. Martin, J. Perkins, D. H. Siegel, R. J. Boucek and I. J. Frieden, Pediatrics, 2013, 131, 128-140.

4 C. Léauté-Labrèze, E. Dumas de la Roque, T. Hubiche, F. Boralevi, J. B. Thambo and A. Taïeb, N. Engl. J. Med., 2008, 358, 2649-2651.

5 K. B. Püttgen, Pediatr. Clin. North Am., 2014, 61, 383-402.

6 A. L. Marqueling, V. Oza, I. J. Frieden and K. B. Puttgen, Pediatr. Dermatol., 2013, 30, 182-191.

7 L. Zhang, H. W. Wu, W. Yuan and J. W. Zheng, Drug Des., Dev. Ther., 2017, 8, 1401-1408.

8 A. Rotter and Z. N. P. de Oliveira, J. Dtsch. Dermatol. Ges., 2017, 15, 1185-1190.

9 Y. Ji, S. Chen, C. Xu, L. Li and B. Xiang, Br. J. Dermatol., 2015, 172, 24-32.

10 L. Zhang, H. M. Mai, J. Zheng, J. W. Zheng, Y. A. Wang, Z. P. Qin and K. L. Li, Int. J. Clin. Exp. Pathol., 2013, 7, 48-55.

11 T. Shimizudani, K. Nagaoka, N. Hanioka, S. Yamano and S. Narimatsu, Chem.-Biol. Interact., 2010, 183, 67-78.

12 Y. Masubuchi, S. Hosokawa, T. Horie, T. Suzuki, S. Ohmori, M. Kitada and S. Narimatsu, Drug Metab. Dispos., 1994, 22, 909-915.

13 P. Partani, Y. Modhave, S. Gurule, A. Khuroo and T. Monif, J. Pharm. Biomed. Anal., 2009, 50, 966-976.

14 G. Tamai, I. Morita, T. Masujima, H. Yoshida and H. Imai, J. Pharm. Sci., 1984, 73, 1825-1827.

15 P. M. Harrison, A. M. Tonkin, C. M. Cahill and A. J. McLean, J. Chromatogr., 1985, 343, 349-358.

16 T. Walle, J. Morrison, K. Walle and E. Conradi, J. Chromatogr., 1975, 114, 351-359.

17 M. W. Lo, B. Silber and S. Riegelman, J. Chromatogr. Sci., 1982, 20, 126-131.

18 Z. F. Jiang, K. L. Shen, Y. Shen and X. Ni, Zhu Futang Practice of Pediatrics, People's Medical Publishing House, Beijing, 2015. 